\title{
A Synchronous and Deterministic MAC Protocol for Wireless Communications on Linear Topologies*
}

\author{
Daniele De Caneva ${ }^{1}$, Pier Luca Montessoro ${ }^{2}$ \\ ${ }^{1}$ Pervasive Technologies Laboratory, Agemont, Italy \\ ${ }^{2}$ Department of Electrical, Managerial and Mechanical Engineering, University of Udine, Udine, Italy \\ E-mail:daniele.decaneva@agemont.it,montessoro@uniud.it \\ Received September 30, 2010; revised October 31, 2010; accepted November 22, 2010
}

\begin{abstract}
Linear topology is useful in several pervasive application scenarios. Even though a linear topology can be handled by unspecific routing algorithms over general purpose MAC protocols, better performance can be obtained by specialized techniques. This paper describes a new communication scheme called Wireless Wire (WiWi), which builds up a bidirectional wireless communication channel with deterministic properties in terms of throughput and latency over a strip of pervasive devices with short-range transmission capabilities. The system is synchronous and fault tolerant. With low cost and extremely simple devices, WiWi builds up a "wire-like" dielectric link, but its applications are not limited to end-to-end communications. For example, WiWi can be used to collect data from sensors along the path, thus acting as a virtual conveyor belt.
\end{abstract}

Keywords: Wireless Sensor Networks, Linear Topology, Pervasive Devices, Ad-Hoc Network

\section{Introduction}

Many routing protocols have been designed for Wireless Sensor Networks (WSNs) considering nodes that operate in a mesh topology. For specific application scenarios, however, a mesh topology may not be appropriate or simply not corresponding to the natural node deployment. Bridge [1] or pipeline [2] monitoring applications are examples where the position of sensor nodes is predetermined by the physical structure and application requirements. In this applications, where it is clearly present a privileged dimension, it is quite natural to take advantage of it.

So far, little focus has been given to efficient MAC protocols for low-power, wireless communications over linear topologies. This paper presents WiWi (Wireless Wire): a contention-free MAC protocol based on synchronous multi-hop transmission along a chain of independent nodes.

The original and main purpose of WiWi is to perform the virtualization of a wired link by means of an ad-hoc network, made of a chain of tiny short-ranged transceivers with limited power capabilities. Nonetheless, WiWi is not limited to end-to-end communication but it can be

"This work has been partially funded by Project A-LEAP/L.R. 26/2005 (art. 21), Regione Friuli Venezia Giulia. profitably used to collect data along the path.

In the WiWi architecture, devices are displaced in order to build up a linear (or curvilinear) strip. WiWi does not require any routing table or complex calculation for message delivery: this makes it feasible to apply the protocol even on very simple transceivers, with limited memory and processing capabilities. Moreover, it is ready for hardware implementations that could be realized on tiny pervasive devices.

The issue regarding synchronization of nodes along the network is addressed by choosing fixed-size messages.

WiWi takes advantage of linear topology and synchronous communication to provide deterministic and predictable latency and throughput in both directions. As will be later described, they can be configured by modifying protocol parameters in order to fulfill the nodes' capabilities and the application requirements.

The paper is organized as follows. After this introduction, Section 2 presents interesting related work. The WiWi architecture, protocol, performance and applications are discussed in Section 3, whereas Section 4 shows the prototypal implementation. A fault-tolerant WiWi node architecture is presented in Section 5, and Section 6 draws some conclusions tracing some expectations for future work. 


\section{Related Work}

Design issues and tradeoffs that need to be considered for power-constrained WSNs with low data rate links have been addressed and studied in noteworthy works [3-5].

A series of studies on routing in ad hoc networks and WSNs face the problem of optimization on behalf of higher layer parameters, such as efficient localization, propagation, resiliency, and so on, proposing a wide variety of algorithmic solutions. Some approaches also examine cross-layer issues that aim at minimizing energy consumption and computation [6]. It is likely to observe that topology in most studies is often in a second order matter, since nodes are expected to be mobile or to be deployed taking random positions in a field. More recently, deployment of wireless sensors has been studied and optimized to achieve coverage and connectivity [7].

Topology is important for any type of network because it has great impact on the performance of the system. Limited research has been conducted on the effect that well-defined topologies have on protocols for wireless networking [8]. The focus, however, has been on mobile networks rather than the ones with regular topologies or with a fixed node placement. The case of patterned WSNs is known in literature and well described in [9].

On the other hand, most works are interested in demonstrating how the topology of a WSN impacts on the performance of a given MAC or routing protocol. Our perspective, instead, aims at simplifying protocol requirements and device complexity starting from a very specific application, in order to propose a reliable and efficient solution for this and similar problems.

Quite common methodologies in WSN-related protocol development [10] recommend that protocols should reduce the number of contentions to improve power saving, as well as using shorter packet lengths. The receiver usage time, however, tends to be higher for protocols that require the mobile nodes to sense the medium before attempting a transmission. In our system, the protocol has been optimized on behalf of these main goals. Moreover, devices' link layer is capable to perform and keep synchronization during the whole lifetime of the network.

In literature we can find some examples of algorithms and protocols that are specifically aimed for linear topologies. MERR [11] is a routing protocol whose reference scenario is a network made by sensors deployed over a linear topology. MERR deals with the problem of finding the best route from every node to a common control center. With MERR, Zimmerling et al. propose a distributed protocol where each node independently chooses the best relay node among its neighbors.

In [12] is presented an algorithm whose aim is to minimize the routing path and, at the same time, balance the load. In particular that work covers the special case of a network where nodes are located in a narrow strip with a width at the most $\sqrt{3} / 2$ times the communications range of each node.

Both [11,12] are focused on routing problems without considering the underlying MAC protocol, which could become a real bottleneck for network performances.

In [13] is presented DiS-MAC (Directional Scheduled MAC). This protocol has been developed for wireless sensor networks that show a linear topology. It reaches the considerable channel utilization of $1 / 2$, but requires every node could direct the radiation beam of its antenna and suffers from being unidirectional. WiWi recalls some DiS-MAC features; in particular both protocols avoid interferences between simultaneous transmissions by alternating transmissions between adjacent nodes. However, WiWi presents many important advantages: it does not require directional antennas, it provides bidirectional communication over a single RF channel (thus providing support for an end-to-end acknowledgement), and it can be configured in order to make the bandwidth and latency fit the application needs.

\section{WiWi Architecture and Communication Protocol}

The development of WiWi was originated by the need for a system able to emulate a wired link by means of an ad hoc network constituted by nodes distributed along a strip. The purpose of this emulation is to handle scenarios where a single hop wireless link is not feasible and a wired link is not practical. An example could be given by a speleologist going deep down into the bowels of the Earth, who can deploy the wireless network while it goes further with the exploration in order to maintain a communication channel with the outside world. Other examples can be found in all those situations where a multihop link is required, in particular those bounded to monitoring applications.

The design of WiWi architecture is based on the observations about wireless communications presented by Min and Chandrakasan in [3]. In particular the authors of [3] showed that even if a power law often describes the radiated power necessary to transmit over a distance $d$ and path loss $n$, this term alone fails to consider the energy overheads of the hardware. Min and Chandrakasan suggested an energy consumption model with an additional distance-independent term in order to take account of overheads caused by transmitter and receiver elec- 
tronics (such as PLLs, VCOs, LNA, bias currents, etc.). The proposed model is the following:

$$
\mathrm{E}(d)=\alpha+\beta d^{n}
$$

where $\alpha$ is the distance-independent term. Additionally, Min and Chandrakasan collected the estimated values of the model's term for a set of short-range radios having maximum output power up to $+20 \mathrm{dBm}$.

Starting from these data, authors of [3] noted that for short-range radios typically used in MANET research, the value of $\alpha$ dominates the value of the path loss term. This has severe consequences on multi-hop wireless communications that try to reduce energy consumption by adding intermediate relay nodes in order to reduce the path loss term. This strategy affects only the path loss term $\left(\beta d^{n}\right)$ limiting the value of $d$, however this is useful only for long range radio links where that term is dominant. The energy model presented in [3] shows that a two hop link consumes less energy than one hop link when

$$
\alpha<\beta d^{n}\left[1-2^{(1-n)}\right]
$$

Min and Chandrakasan noted that with the exception of the $\mu$ AMPS-1 custom radio, this inequality never holds for typical path losses.

Similar considerations where made in [14] where Bhardwaj et al. introduced the concept of characteristic distance of a transceiver. This distance is strictly bounded to the transceiver characteristics and minimizes power consumption in a multi-hop communication. In fact, the characteristic distance represents the optimal tradeoff between distance-independent and distance-dependent terms in power consumption relation.

WiWi has been designed as a synchronous multi-hop communication scheme where nodes are intended to be deployed with mutual distances approximately close to the characteristic distance (Figure 1). No other assumption is made over node deployment: the chain of nodes is not required to strictly follow a straight path as it can bend. Moreover, in order to minimize the possible production costs, we assumed that pervasive devices could be absolutely identical one to each other, in the sense that no factory-set unique ID is required.

A synchronous architecture is not very common in typical sensor networks described in literature, nevertheless it is our opinion that its deterministic behavior better suites the aim of WiWi.

A detailed description of the synchronous communica- tion pattern is presented in the following subsection.

\subsection{Basic Communication Pattern}

The communication between WiWi nodes is synchronous, based on fixed size packets, and follows a staggered pattern like the model presented in DMAC [15]. However, unlike the DMAC protocol, which is designed to handle tree topologies, in WiWi the synchronism is intrinsic in the communication model and bidirectional data flows are supported.

Figure 2 shows the communication model and the different handling of downstream and upstream data flows. In this and following diagrams the vertical axis is depicted downward, according to the direction of the downward flow that provides synchronization to all the nodes. The master node, source of synchronization, is at position $\mathrm{n}=0$.

The downstream data flow is generated by the head of the chain which acts as master end point. This data flow (the gray one in Figure 2) proceeds downwards from the head of the chain to the tail following a strictly staggered pattern: a node sends a packet to the next one, which in turn immediately forwards the packet further down along the chain. This stream is responsible of maintaining overall network synchronization too: every node resynchronizes its clock upon the start of the incoming downstream packets.

The upstream flow follows the same principle of passing messages along the chain, but between the reception of a packet and its forwarding, the node waits four time slots in order not to collide with the downstream one. In Figure 2, the upstream flow is depicted in white blocks, while the arrows of different patterns follow the advancement of different upstream packets.

This scheme combines very good performance with high regularity that makes its implementation easy. In fact, once a node is synchronized with the downstream flow, its activity pattern is receive-transmit-idle-transmitreceive-idle (R-T-I-T-R-I) regardless its position in the chain.

Moreover, in WiWi, nodes require no explicit addressing because within the range of transmission there is only one receiving node, i.e. the destination of the next hop for the packet.

It is worth noting that the transmission scheme is designed to avoid interferences among different nodes. In

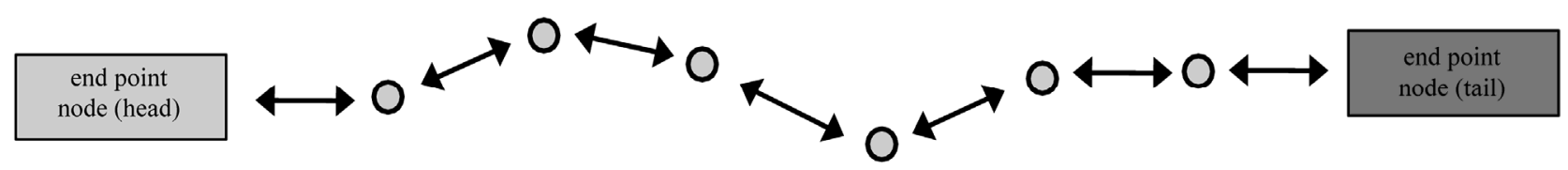

Figure 1. WiWi topology: The linear strip as a chain of nodes. 


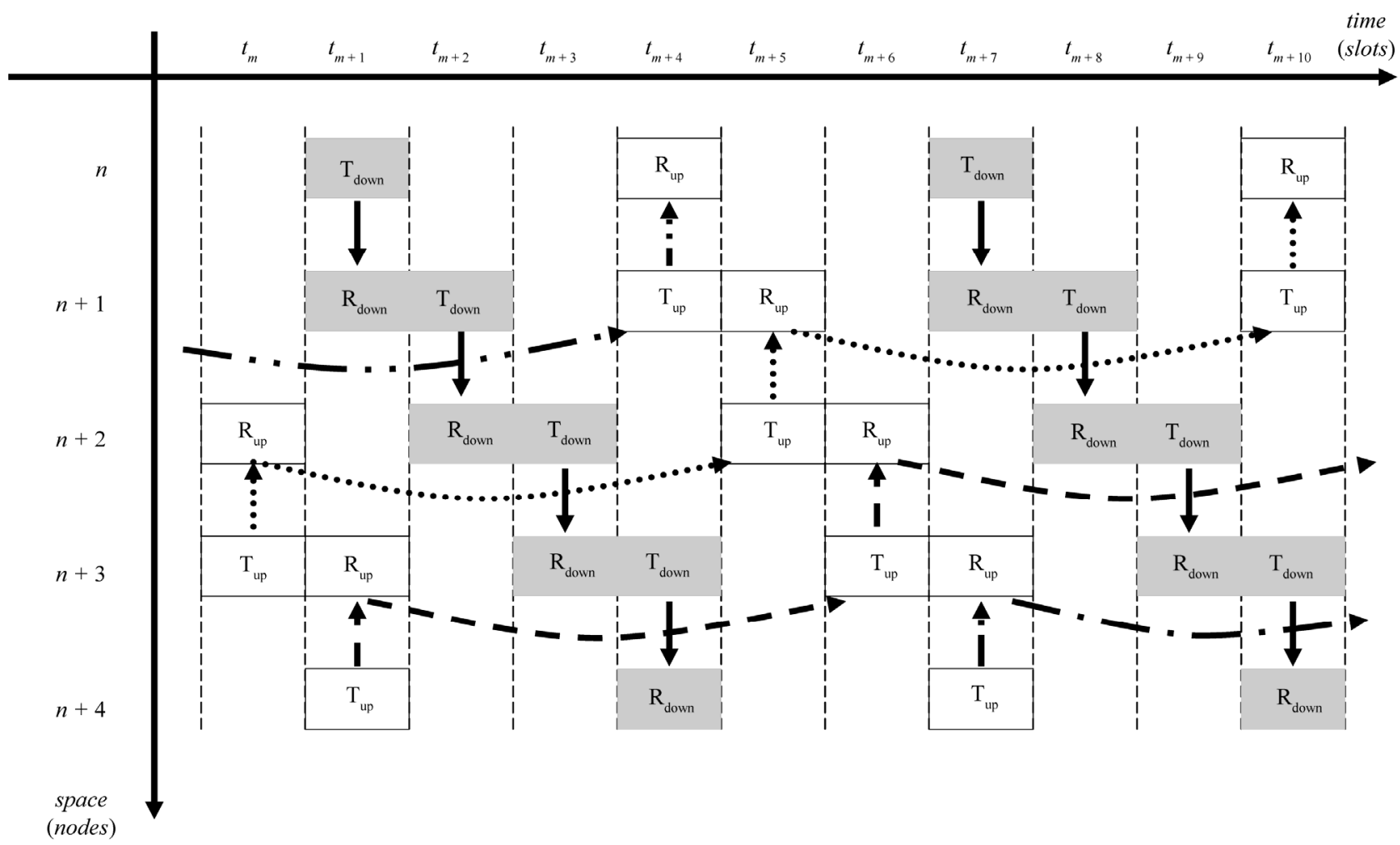

downstream packets

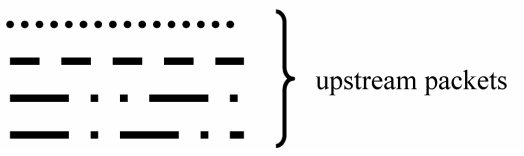

Figure 2. Bidirectional staggered transmission.

fact, from the receiver point of view, when an adjacent node is transmitting, the closest other transmitting node is three hops away. So, considering the common assumption that the interference radius is twice the nominal transmission one [13] this three-hop safe distance grants a good resiliency from inter-node interference.

\subsection{Performance Evaluation and Advanced Patterns}

A performance evaluation of this scheme of the communication pattern is straightforward. Let $N$ be the number of hops of the link, $B$ the maximum raw bit rate and $\mathrm{S}$ the time slot associated with a single packet transmission (which will also include a communication safety time in order to handle clock drifts between consecutive nodes). Both downstream and upstream flows use one time slot every six, therefore each one provides a bit rate of nearly $B / 6$ and the overall channel utilization is equal to $1 / 3$ (1/6 for each stream). Actually, the bit rate will necessarily be lower than $B / 6$ due to non-idealities of hardware (e.g. clock drifts). It is worth noting that a channel utilization equal to $1 / 3$ is the optimum for a bidirectional commu- nication, being the maximum allowed by the minimum three-hops safe distance chosen to avoid interference between simultaneous transmissions. Additionally, the throughput of both streams equal to $1 / 6$ of the transceiver rate has to be considered a good tradeoff between performance and latency, since in [16] the maximum operational limit of a unidirectional wireless chain is called to be equal to $1 / 4$.

The downstream flow has the minimum latency allowed by a store-and-forward pattern, being the delay equal to $N \cdot S$. On the other hand the upstream flow delay is five times larger: since a packet can be sent every six time slots, there is, in average, an additional constant delay of three time slots per hop.

However, WiWi architecture is flexible: the receivetransmit pattern can be adjusted to fit the desired tradeoff between latency, bandwidth and symmetry of upstream and downstream flows, at the cost of a lessthan-optimal channel utilization. Even the distance between simultaneously active transmitters could be changed depending on the RF layer requirements. Figure 3 shows a pattern similar to the previous one but providing asymmetrical throughput $(B / 5$ downstream and $B / 10$ 

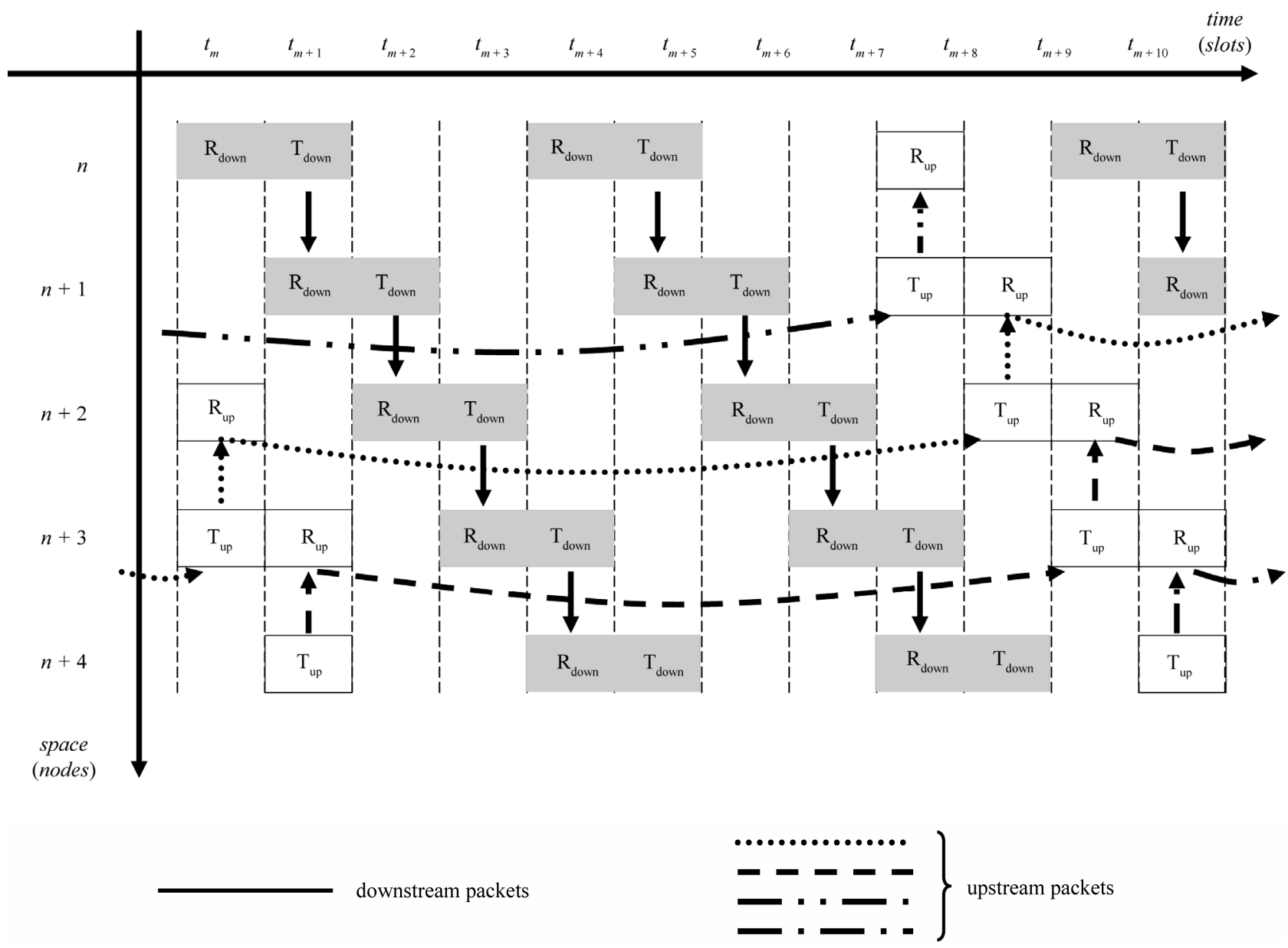

Figure 3. Bidirectional, staggered transmission with asymmetric throughput and latency over a WiWi link.

upstream) and asymmetrical latency $(N \cdot S$ downstream and $10 \mathrm{~N} \cdot \mathrm{S}$ upstream, plus in average 2.5 time slots and 5 time slots respectively to wait for the transmission window).

Figure 4 shows another pattern where both bandwidth and latency are symmetrical: a transmission occurs every eight time slots in each direction, providing an overall channel utilization of $25 \%$, whereas the latency is $2 N \cdot S$. The block arrows show the travel, in space and time, of downstream and upstream packets.

Unfortunately, in this case the receive (R), transmit (T) and idle (I) pattern is no longer independent from the cluster position along the chain: even-order clusters run R-R-T-T-I-I-I-I, whereas odd-order clusters behave R-I-T-R-I-T-I-I, thus requiring a bit more complex chain setup.

WiWi does not provide acknowledgment to guarantee the correct packet exchange between nodes: datagram transmission is much more flexible in the scenarios in which WiWi is supposed to operate. This choice implies that the nodes could be very simple because they do not have to store more than two packets at a time in their internal memory. Anyhow, error correction codes could be adopted to increase the reliability of communications along the WiWi chain. In addition, an acknowledgement system could be implemented by higher layer protocols between the head and the tail of the strip. In scenarios where the end points are many hops away from each other and the packet error rate is particularly severe, this solution could be responsible of poor latency performance due to frequent retransmissions. This problem could be mitigated by displacing intermediate endpoints, which corresponds to deploy many WiWi strips in sequence instead of one single strip.

\subsection{Strip of Sensors: WiWi as a Conveyor Belt}

So far, WiWi has been presented as a protocol for end-to-end communication on a strip of short-range wireless devices. But the WiWi synchronous nature makes it a powerful tool to collect data from a strip of sensors. Conventionally, in routing-oriented sensor networks, a sensor places its reading in a packet that finds its way through the network, fulfilling at each hop the 


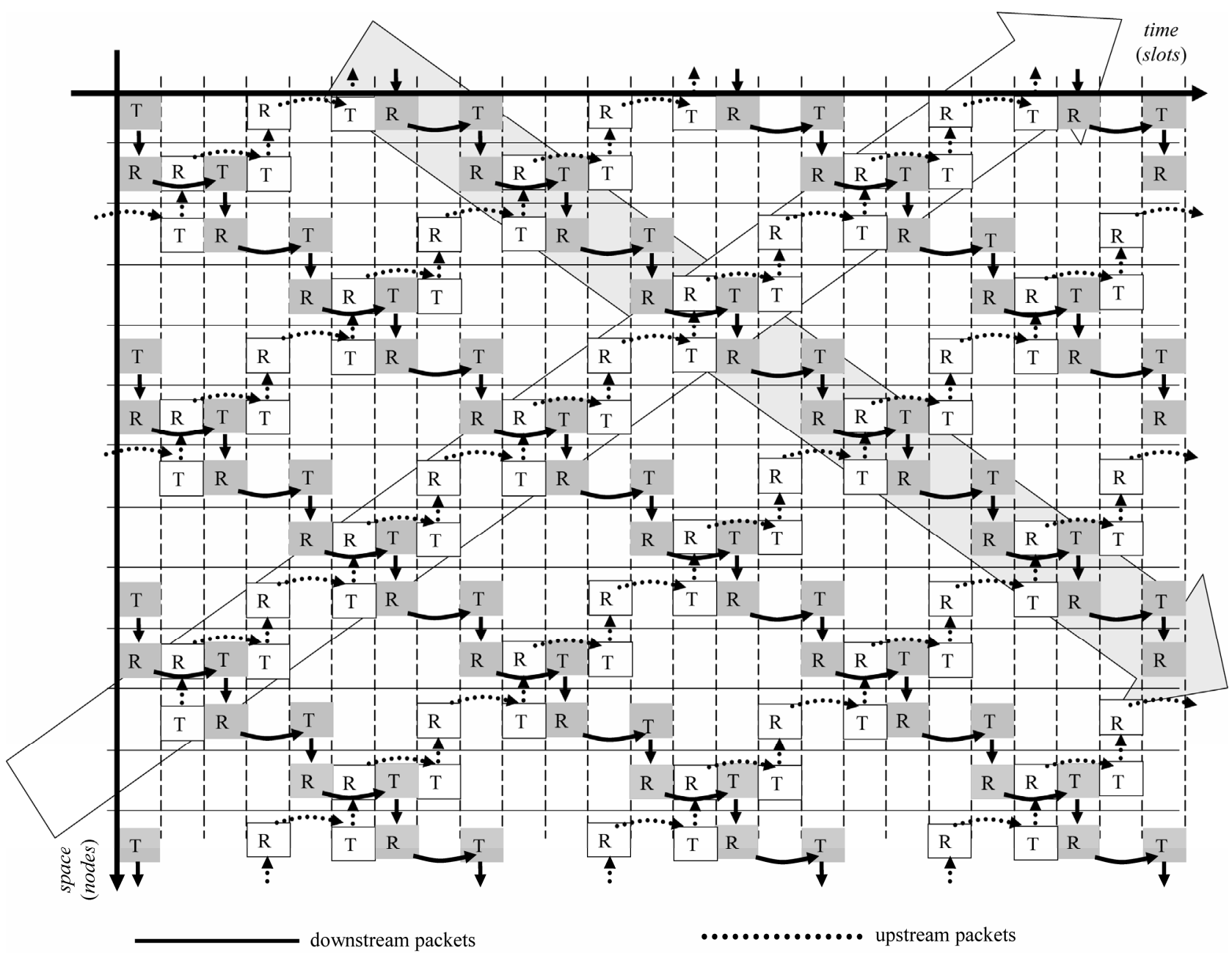

Figure 4. Bidirectional, staggered transmission with symmetric throughput and latency over a WiWi link.

chosen MAC rules. In WiWi each node can host one or more sensors. The data flow that continuously traverses the strip can be seen as a conveyor belt on which the sensors place their readings. Several application-level protocols can be adopted to handle possible conflicts: for example, a flag that marks each time slot when vacant, or a more sophisticated token-based protocol.

The WiWi application as conveyor belt for a strip of sensor has been tested using accelerometers in our prototypal implementation, as described in the next section.

\section{Testing and Prototyping}

WiWi has been validated in both fault-free and faulty scenarios (see Section 5) by simulation using the Omnet++ platform [17], but the obtained results are limited by the validity of the transmission model that, in the real world, should include RF hardware performance, battery levels, environment, interferences, etc.

In order to verify the feasibility of the proposed ap- proach and show the effectiveness of the system, we developed a prototype for two demo applications: end-to-end communications and strip of sensors.

The prototype (Figure 5) accommodates a Microchip microcontroller (PIC16F689), I/O hardware for debugging purposes, and a transceiver. Two different transceiver modules by Aurel [18] have been tested; their bit rates and transmission ranges are reported in Table $\mathbf{1 .}$

Table 1. Transceivers performance.

\begin{tabular}{|c|c|c|c|}
\hline Transceiver & Modulation & Bit rate & $\begin{array}{c}\text { Transmission } \\
\text { range }\end{array}$ \\
\hline $\begin{array}{c}\text { XTR-CYP-2 } \\
.4 \mathrm{GHz}\end{array}$ & $\begin{array}{c}\text { GFSK, } \\
78 \text { channels } \\
2.4 \mathrm{GHz} \text { band }\end{array}$ & $\begin{array}{c}\text { up to } \\
64 \mathrm{Kbit} / \mathrm{s}\end{array}$ & $45 \mathrm{~m}$ \\
\hline $\begin{array}{l}\text { XTR-VF- } \\
2.4 \text { LP }\end{array}$ & $\begin{array}{c}\text { GFSK, } \\
98 \text { channels } \\
2.4 \mathrm{GHz} \text { band }\end{array}$ & $\begin{array}{l}\text { up to } \\
1 \mathrm{Mbit} / \mathrm{s}\end{array}$ & $10 \mathrm{~m}$ \\
\hline
\end{tabular}




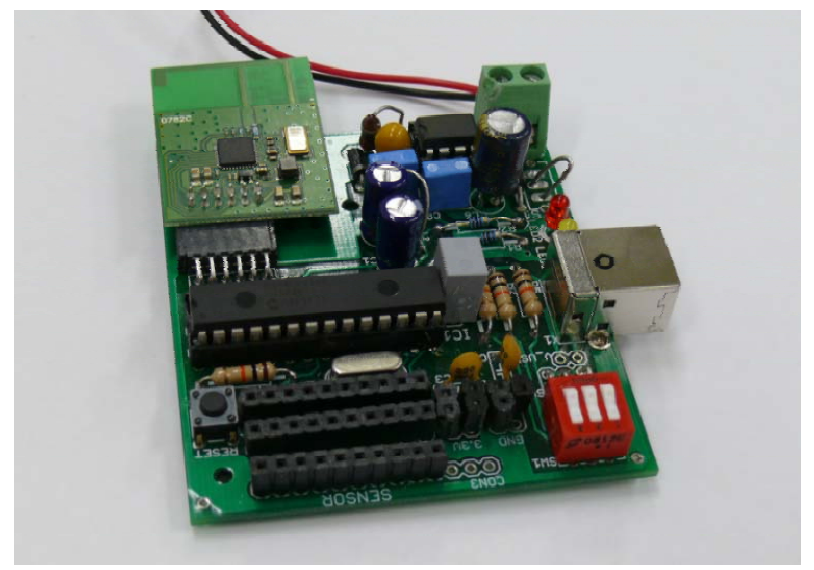

Figure 5. Hardware prototype $(5 \mathrm{~cm} \times 6 \mathrm{~cm})$.

The prototype is equipped with a switching step-up voltage regulator in order to reduce power consumption and make possible the use of two AA batteries as power supply. USB connectivity has been included in order to expedite firmware debugging and enable WiWi endpoints to exchange data with any common PC.

The first application developed with this prototype emulated a chat application between two computers, using WiWi as channel to deliver messages from one user to the other (Figure 6).

In the second application WiWi has been used to aggregate and transport data coming from 3-axis accelerometer sensors connected to WiWi nodes (Figure 7). Data coming from sensors were preprocesses by nodes in order to filter noise and obtain the maximum acceleration for each axis in 10 milliseconds time windows. Since this kind of application doesn't need an extremely high throughput, $500 \mathrm{~ms}$ time slots have been used, much longer that the $1 \mathrm{~ms}$ packet length. This way proper throughput and consumption performance have been achieved since each node had more time to spend in low power mode. At each step along the chain, data measurement pertaining to the traversed node was inserted into a specific field within the current packet. Since this data-collecting application is essentially unidirectional, the downstream flow was delegated to remote control and management of the nodes. This flow was much less intense, therefore downstream packets were shorter (one quarter of upstream packets), resulting in additional energy saving, since idle time has been further increased.

\section{Designing a Fault-Tolerant WiWi Node}

An obvious weakness of chain topologies is that a single node failure can block the whole communication channel. Nevertheless, if the topology of the physical environment supposed to host the chain is strictly linear, neither backup paths nor meshed topologies can be deployed. Since

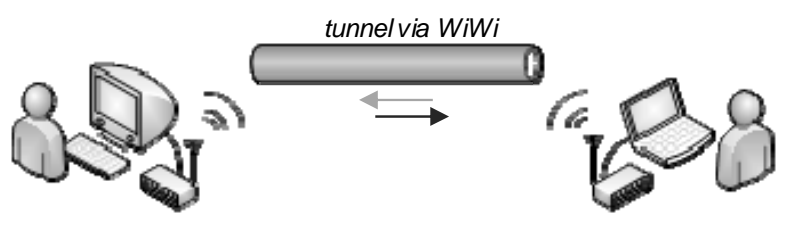

Figure 6. Chat demo application.
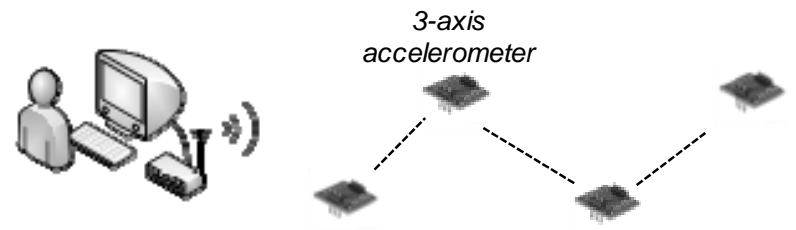

Figure 7. Sensoristic demo application.

multiple nodes within the same transmission range are not suitable for the WiWi synchronous protocol due to interferences and performance loss, a fault-tolerant WiWi node has been designed.

A fault tolerant WiWi node is made of multiple transceiver modules (Figure 8), each one able to handle the whole communication protocol. Their activity is scheduled on behalf of a round robin policy. Just one module at a time is effectively involved in packet forwarding, while the other ones act as backup modules. The modules are ordered, and the first one is the module on duty, responsible for packet forwarding.

During the receive time slot every module receives and stores the packet coming from the previous node in the chain. In the subsequent transmission slot, the module on duty forwards the packet, while at the same time all backup modules sense the transmission to be sure it occurs. If the first backup module perceives the loss of the module on duty, after a short "sense time slot" it forwards the packet. The remaining backup modules keep listening to the channel in order to be sure that a backup module has reacted. The process is iterated until a backup module forwards the packet or there are no more backup modules left. Figure 9 shows this technique giving an example of redundancy and failure management over four modules: at the beginning of the transmit slot the module on duty (number 0 ) should forward the packet (time $t_{1}$ ). Modules 1, 2 and 3 start sensing the transmission for the duration of the $s_{1}$ sensing window. If no transmission occurs, at time $t_{2}$ node 1 should backup module 0 transmitting the packet. Remaining modules 2 and 3 continue to sense, in order to backup module 1 (with module 2 at time $t_{3}$ ) and, if still needed, module 2 (with module 3 at time $t_{4}$ ). In the example the packet is forwarded by the last backup module.

After a module failure, active backup modules redefine their order. This way, the same technique can be used to provide fault tolerance and to schedule sleep-times 


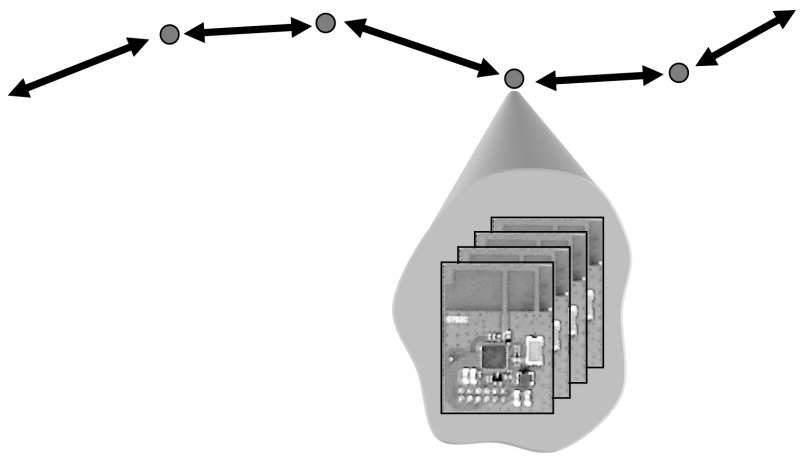

Figure 8. Hardware prototype.

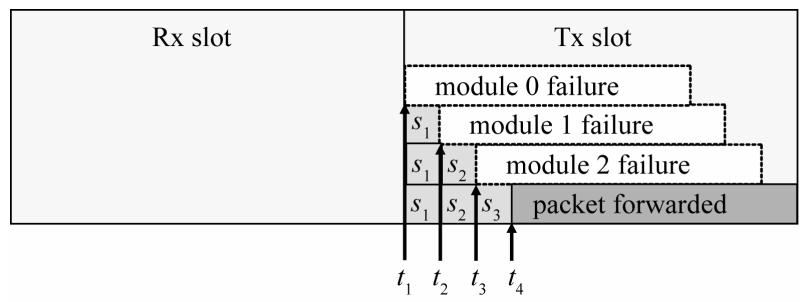

Figure 9. Redundancy mechanism.

for load balancing and energy saving.

This fault tolerance extension to the basic WiWi protocol grants a redundancy equal to the number of backup modules. The slot time upon which WiWi is based must be extended to make room to the sensing time windows, leading to a minor loss in throughput and latency performances.

The extended time slot can be modeled as in Figure 10. Being $N_{\max }$ the maximum number of nodes belonging to a pool, the duration of a slot is represented by three components:

- $T_{P}$ : time needed to receive the packet, depending only on packet size and transceiver bit-rate;

- $T_{S}$ : sensing time window: sum of the sensing time and the time needed to commute the radio module from sensing state to transmitting state;

- $T_{G}$ : the inter-packet gap, a period comprehensive of the time needed to switch from the receiving state to the transmitting state, the time needed to store and elaborate the packet, and a safety margin to counteract clock drifts.

Hence, with $N_{\max }$ modules in each node, the time dedicated to packet transmission is equal to $T_{S}\left(N_{\max }-1\right)+T_{P}$ and the total duration $T$ of a slot is:

$$
T=T_{P}\left(N_{\max }-1\right)+T_{P}+T_{G}
$$

Thanks to the decoupling of the tiers composing the architecture, the other performance relations remain valid. In fact throughput is equal to $T_{p c k} /\left(6 T_{S}\right)$ while latency is equal to $N T_{S}$ for the downstream flow and $(N+3) T_{S}$ for the upstream flow.

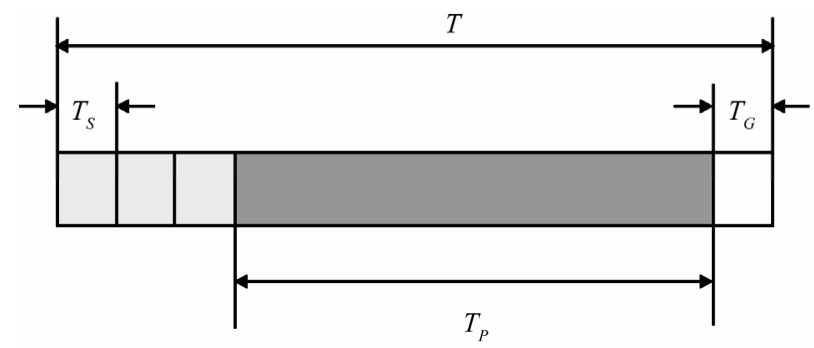

Figure 10. Time slot components.

The backup technique requires modules within each pool to be dynamically enumerated and ordered. Optionally, the architecture could include a set of algorithms for modules deployment and management that do not require any manual configuration or the assignment of unique node ID at firmware/hardware level. This aspect is not discussed here because several algorithms for cluster organization can be found in literature and easily applied to WiWi.

\section{Conclusions and Future Work}

A new MAC protocol for linear topologies, called WiWi, has been presented. It is based on a chain of short-range communication devices. Predictable latency and bandwidth are provided by the synchronous communication scheme and by specific transmit-receive patterns that have been shown in Section 3. Fault tolerant node design has been discussed as well. WiWi has been simulated and tested on hardware prototypes.

Strategies to use WiWi in different scenarios are currently under study, in particular to use the strip as a fundamental brick for more complex topologies. Moreover, different channels can be used to handle overlapping strips, thus allowing a variety of topologies that combines TDMA and FDMA techniques. In tree topologies, an asymmetric configuration of the protocol can be exploited to benefit the links from the root to the leaves, making WiWi a challenging solution to control and deliver data to a large number of devices in widespread applications.

As a synchronous technique, WiWi is well suitable to include strategies, already known in literature, to schedule stand-by periods and wake-up events to save energy. Since each strategy may be optimal only for one or few kind of applications, another current research direction is to define some detailed application scenarios and select for them the optimal energy-saving schemes.

\section{Acknowledgements}

Authors wish to thank Giampietro Tecchiolli from Eurotech S.p.A. for his encouragements and his interest in 
WiWi application scenarios. We also wish to thank Francesco Cossettini and Manuel Nobile for their contribution in the simulations of the protocol and the development of the hardware prototype.

\section{References}

[1] S. Kim, S. Pakzad, D. Culler, J. Demmel, G. Fenves, Glaser and M. Turon, "Health Monitoring of Civil Infrastructures Using Wireless Sensor Networks,” Proceedings of 6th International Symposium on Information Processing in Sensor Networks, Cambridge, 25-27 April 2007, pp. 254-263.

[2] I. Jawhar, N. Mohamed and K. Shuaib, “A Framework for Pipeline Infrastructure Monitoring Using Wireless Sensor Networks," Proceedings of Wireless Telecommunications Symposium, Pomona, 26-28 April 2007, pp. 1-7.

[3] R. Min and A. Chandrakasan, "Top Five Myths about the Energy Consumption of Wireless Communication,” ACM SIGMOBILE Mobile Computing and Communications Review, Vol. 7, No. 1, 2003, pp. 65-67.

[4] A. Youssef, J. Haslett and S. Magierowski, "Design Issues for Sensor Network RF Receivers,” Proceedings of Canadian Conference on Electrical and Computer Engineering, Vancouver, 22-26 April 2007, pp. 1535-1538.

[5] C. Schurgers, V. Tsiatsis, S. Ganeriwal and M. Srivastava, "Optimizing Sensor Networks in the Energy-LatencyDensity Design Space,” IEEE Transactions on Mobile Computing, Vol. 1, No. 1, 2002, pp. 70-80.

[6] Y. Zhang and L. Cheng, "Cross-Layer Optimization for Sensor Networks," Proceedings of New York Metro Area Networking Workshop, New York, September 2003, pp. 1-3.

[7] X. Bai, S. Kuma, D. Xua, Z. Yun and T. H. La, "Deploying Wireless Sensors to Achieve both Coverage and Connectivity," Proceedings of 7th ACM International Symposium on Mobile Ad Hoc Networking and Computing, Florence, 22-25 May 2006, pp. 131-142.

[8] A. Conti and D. Dardari, "The Effects of Nodes Spatial Distribution on the Performance of Wireless Sensor Networks," Proceedings of IEEE 59th Vehicular Technology Conference, Milan, Vol. 5, 17-19 May 2004, pp. 2724-
2728.

[9] H. M. F. AboElFotoh, E. S. El Mallah and H. S. Hassanein, "On the Reliability of Wireless Sensor Networks," Proceedings of IEEE International Conference on Communications, Istanbul, Vol. 8, 11-15 June 2006, pp. 34553460.

[10] J. Chen, K. M. Sivalingam and P. Agrawal, "Performance Comparison of Battery Power Consumption in Wireless Multiple Access Protocols," Wireless Networks, Vol. 5, No. 6, 1999, pp. 445-460.

[11] M. Zimmerling, W. Dargie and J. M. Reason, "EnergyEfficient Routing in Linear Wireless Sensor Networks," Proceedings of IEEE International Conference on Mobile Adhoc and Sensor Systems, Pisa, 8-11 October 2007, pp. $1-3$.

[12] L. Zhang and J. Gao, "Load Balanced Short Path Routing in Wireless Networks,” IEEE Transactions on Parallel and Distributed Systems, Vol. 17, No. 4, April 2006, pp. 377-388.

[13] T. Karveli, K. Voulgaris, M. Ghavami and A. H. Aghvami, "A Collision-Free Scheduling Scheme for Sensor Networks Arranged in Linear Topologies and Using Directional Antennas," Proceedings of 2 nd International Conference on Sensor Technologies and Applications, Cap Esterel, 25-31 August 2008, pp. 18-22.

[14] M. Bhardwaj, T. Garnett and A. Chandrakasan, "Upper Bounds on the Lifetime of Sensor Networks," Proceedings of IEEE International Conference on Communications, Helsinki, 11-14 June 2001, pp. 785-790.

[15] G. Lu, B. Krishnamachari and C. S. Raghavendra, “An Adaptive Energy-Efficient and Low-Latency MAC for Data Gathering in Sensors Networks," Proceedings of 18th IEEE International Parallel and Distributed Processing Symposium, Santa Fe, 26-30 April 2004, pp. 224231.

[16] J. Li, C. Blake, D. Couto, H. Lee and R. Morris, "Capacity of Ad Hoc Wireless Networks," Proceedings of the 7th ACM Annual International Conference on Mobile Computing and Networking, Rome, 16-21 July 2001, pp. 61-69.

[17] Omnet++ Community, 2010. http://www.omnetpp.org

[18] Aurel S.p.A., 2010. http://www.aurel.it 